\title{
General analytical solutions for DC/AC circuit-network analysis
}

\author{
Nicolás Rubido $^{1, a}$, Celso Grebogi ${ }^{2, b}$, and Murilo S. Baptista ${ }^{2, c}$ \\ 1 Universidad de la República, Facultad de Ciencias, Iguá 4225, Montevideo, Uruguay \\ 2 University of Aberdeen, King's College, Institute for Complex Systems and \\ Mathematical Biology, AB24 3UE Aberdeen, UK
}

Received 4 April 2017

Published online 21 June 2017

\begin{abstract}
In this work, we present novel general analytical solutions for the currents that are developed in the edges of network-like circuits when some nodes of the network act as sources/sinks of DC or $\mathrm{AC}$ current. We assume that Ohm's law is valid at every edge and that charge at every node is conserved (with the exception of the source/sink nodes). The resistive, capacitive, and/or inductive properties of the lines in the circuit define a complex network structure with given impedances for each edge. Our solution for the currents at each edge is derived in terms of the eigenvalues and eigenvectors of the Laplacian matrix of the network defined from the impedances. This derivation also allows us to compute the equivalent impedance between any two nodes of the circuit and relate it to currents in a closed circuit which has a single voltage generator instead of many input/output source/sink nodes. This simplifies the treatment that could be done via Thévenin's theorem. Contrary to solving Kirchhoff's equations, our derivation allows to easily calculate the redistribution of currents that occurs when the location of sources and sinks changes within the network. Finally, we show that our solutions are identical to the ones found from Circuit Theory nodal analysis.
\end{abstract}

\section{Introduction}

The currents in each edge of an electrical circuit, which is composed of linear elements (i.e., resistance, capacitance, and inductance) and where conservation of charge at each node is granted, are generally found by solving Kirchhoff's equations [1]. In particular, for resistor networks, the solution for the currents at each edge is related to random walks in graphs [2], first-passage times [3], finding shortest-paths and community structures on weighted networks [4], and network topology spectral

\footnotetext{
a e-mail: nrubido@fisica.edu.uy

b e-mail: grebogi@abdn.ac.uk

c e-mail: murilo.baptista@abdn.ac.uk
} 
characteristics [5,6]. Although the relationship between currents and voltage differences follows Ohm's law, network circuits modelling capabilities are enormous. For example, circuit networks are used to model fractures in materials [7], biologically inspired transport networks [8], airplane traffic networks [9], robot path planing [10], queueing systems [11], to name a few.

In practice, resistor networks are used in various electronic designs, such as current or voltage dividers, current amplifiers, or digital to analogue converters. These devices are usually inexpensive, relatively easy to manufacture, and require little precision on the constituents. In order to solve the voltages across these networks, two methods are broadly used: nodal analysis and mesh analysis [1]. In the former, nodes are labelled arbitrarily and voltages are set by using Kirchhoff's current equations. In the later, loops are defined with an assigned current, discarding any inner loops; then, Kirchhoff's voltage equations are solved. These constitute classic techniques of Circuit Theory.

However, nodal and mesh methods (or even transfer function methods [12]) become inefficient to recalculate the voltage drops across the network if the location of inputs and outputs changes constantly, e.g., if the cathode and/or anode of a voltage generator are moved from one node of the network to another. This switching situation is common in the modelling of the modern power-grid as an impedance network circuit or in general supply-demand networks [5-8]. An example of this case is shown in Figure 1 for a resistor network with a single source-sink nodes. Another redistribution of currents, which is also poorly accounted by these methods, happens if a single source node and single sink node are decentralized for multiple sources and/or multiple sinks that preserve the initial input and output magnitudes $[7,13]$. In any case, both former situations require the application of the classical Circuit Theory methods for each configuration of the sources and sinks in order to find the currents at every edge of the network.

In this work, we present general solutions for current conservative $\mathrm{DC} / \mathrm{AC}$ circuit networks with resistive, capacitive, and/or inductive edge characteristics. The novelty in our derivations comes from expressing the currents and voltage drops in terms of the eigenvalues and eigenvectors of the admittance (namely, the inverse of the edge impedance) Laplacian matrix of the circuit network.

In order to derive our solutions we assume that the impedance values at every edge and the location of the source/sink nodes are known. Our solutions give the exact $\mathrm{DC} / \mathrm{AC}$ currents that each edge of the circuit holds under these conditions and are identical in magnitude to the ones found from nodal Circuit Theory analysis. The practicality of our solutions comes from, allowing to compute the equivalent impedance between any two nodes of the network directly [14-16] and allowing to easily calculate the redistribution of currents that happens when the location of sources and sinks is changed within the network (such as in the example of Fig. 1). In this sense, we are performing a direct calculation of Thévenin theorem [17] without the need for short-circuiting the system.

The scientific interest of our solutions comes from establishing a clear relationship between the currents and voltages in $\mathrm{DC} / \mathrm{AC}$ circuits with the topology invariants of the network. In other words, it provides a rigorous link between network topology and both the steady-state constant (DC) and oscillatory (AC) currents of a conservative circuit network. Moreover, the approach we develop provides new analytical insight into the transmission flow problem [18], exhibiting different features than other available solutions, and allows to analyse resonant behaviour in linear circuits [19]. Previous studies about dynamics and topology informed on the intertwined relationship that these observables hold. In particular, when a network of equal oscillators becomes fully synchronous in their amplitudes and phases, the eigenvalues of the Laplacian matrix (topology) provide bounds for the coupling strength of the oscillatory modes (dynamics) to become stable [20-22]. In our approach, the nodes are 
(a)

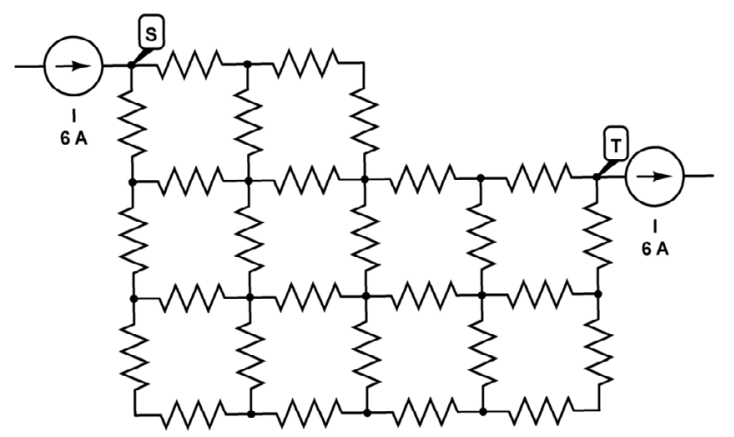

(b)

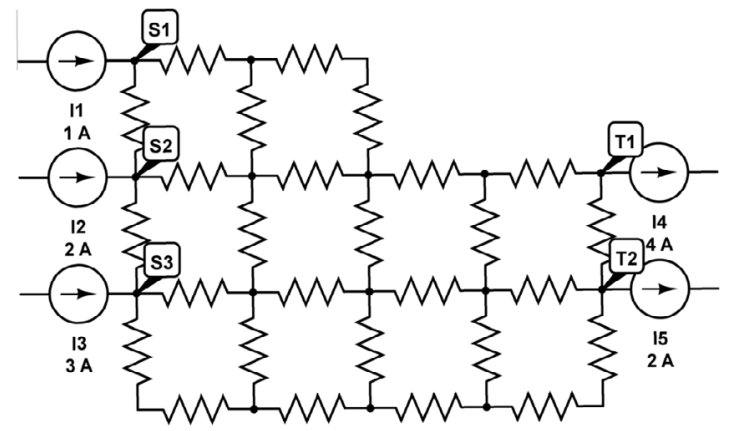

Fig. 1. Panel (a) shows a schematic representation of a resistor network with a single input current of $I=6 \mathrm{~A}$ at a source node $S$ and a single output current of $I=6 \mathrm{~A}$ at a sink node $T$. Panel (b) shows the same resistor network but with multiple inputs $I_{1}, I_{2}, I_{3}$ (nodes $S_{1}, S_{2}, S_{3}$ ) and outputs $I_{4}, I_{5}$ (nodes $T_{1}, T_{2}$ ) which add to the same inflow/outflow magnitudes than in panel (a). Changing the system from panel (a) to panel (b), or vice-versa, generates a global redistribution of currents.

not considered to be amplitude synchronous since they are passive entities, however our solutions provide an extension and a compelling link between topology and the steady-state dynamic in complex structures. Our DC/AC circuit-network analysis provides a solution other than the one from Kirchhoff's analysis. At the same time, it also provides solutions to two paradigmatic broadly used non-linear models of complex system: the random walker moving along an arbitrary complex structure (the DC solution) and the generalised Kuramoto phase-oscillator (i.e., the second order Kuramoto model) network in its steady-state solution (AC solution). As a practical application, we relate these solutions to closed circuits where a voltage generator is present (instead of having open sources/sinks that feed current to the network) and solve a simple network where we can compare our solutions to the ones provided by solving directly Kirchhoff's nodal equations.

\section{$2 \mathrm{DC} / \mathrm{AC}$ circuit networks}

\subsection{The mathematical model}

The model we solve corresponds to a conservative circuit network with known input/output net currents and obeys Ohm's and Kirchhoff's law for conservation of 
charge. We assume that the input (output) net current $\sum_{k} I_{s k}\left(\sum_{k} I_{t k}\right)$ at the source (sink) node $s(t)$, its frequency $\omega$ (with $\omega=0$ for DC currents and $\omega>0$ for AC currents), and their phases are known. The extension to various input or output nodes is done in Appendix C.

Ohm's law linearly relates the current at an edge of the circuit with the voltage difference between the nodes that the edge connects. Specifically,

$$
I_{k l}^{(s, t)}=\frac{V_{k}^{(s, t)}-V_{l}^{(s, t)}}{Z_{k l}}=\frac{\Delta V_{k l}^{(s, t)}}{Z_{k l}},
$$

where $I_{k l}^{(s, t)}$ is the current passing from node $k$ to node $l$ given a current source located at node $s$ and a sink node located at node $t, \Delta V_{k l}^{(s, t)}$ is the voltage difference, and $Z_{k l}=Z_{l k}$ is the impedance of the symmetric edge. $Z_{k l}$ depends on the edge's resistive, capacitive, and/or inductive properties, and the network topological properties of the circuit.

The variables in equation (1) are complex numbers in the case of $\mathrm{AC}$ input/output currents and real numbers for DC currents. In general, a resonance in the $k l$-edge appears for a minimum of the impedance, namely, when the input/output frequency $\omega$ is tuned to a frequency related to the natural frequency of the edge line. For example, in the case that the edge is modelled by a series $R L C$ circuit, the impedance of the edge is

$$
Z_{k l}=R_{k l}+j \omega L_{k l}\left[1-\left(\frac{\omega_{k l}^{(L C)}}{\omega}\right)^{2}\right]=Z_{l k}
$$

where $\omega_{k l}^{(L C)}=\sqrt{1 / L_{k l} C_{k l}}$ is the natural frequency of the edge, $\gamma_{k l}^{(R L)}=R_{k l} / L_{k l}$ is the dissipation of the edge, $L_{k l}$ is the edge's inductance, $C_{k l}$ is the edge's capacitance, $R_{k l}$ is the edge's resistance, and $j=\sqrt{-1}$. In this case, a resonance in the $k l$-edge appears if $\omega=\omega_{k l}^{(L C)}$. Consequently, our solutions are valid as long as the input/output frequency $\omega$ is different from any of the $M$ resonant frequencies associated to the $M \in[N-1, N(N-1) / 2]$ edges of the connected circuit network.

The topological properties of the network are also included in the value of the impedance of each edge. For instance, the impedance between two nodes that are disconnected is assumed to be $\left|Z_{k l}\right|=\infty$ because $R_{k l}=\infty$ for a non-existing line. Otherwise, the impedance of two connected nodes is $\left|Z_{k l}\right|<\infty$. Hence, the inverse of the impedance, which is known as the admittance, defines a complex valued matrix, giving the edges complex weight connecting each pair of nodes in the circuit.

Kirchhoff's charge conservation law states that current is conserved at every node in the circuit. In other words, the inflow equals the outflow at a node, with the exception of the source and sink nodes (the extension to multiple sources and sinks is detailed in Appendix C). Specifically,

$$
\sum_{l=1}^{N} I_{k l}^{(s, t)}=\delta_{k s} F^{i n}-\delta_{k t} F^{o u t}
$$

where $F^{\text {in }}\left(F^{\text {out }}\right)$ is the complex valued net current inflow (outflow) and $\delta_{k s}\left(\delta_{k t}\right)$ is the Kronecker delta function. By assuming local conservation of charge at every node of the circuit, we get that the total flow in the network needs to be null, namely, global conservation of charge holds: $\sum_{k} \sum_{l} I_{k l}^{(s, t)}=0$, which is fulfilled only if $F^{i n}=$ $F^{\text {out }}=F$. 
Using equations (1) and (3), the model equations are

$$
V_{k}^{(s, t)} \sum_{l=1}^{N} \frac{1}{Z_{k l}}-\sum_{l=1}^{N} \frac{V_{l}^{(s, t)}}{Z_{k l}}=F\left(\delta_{k s}-\delta_{k t}\right),
$$

which are expressed in matrix form as

$$
\mathbf{G} \mathbf{V}^{(s, t)}=\mathbf{F}^{(s, t)},
$$

where $\mathbf{G}$ is the weighted admittance Laplacian matrix of the network [with complex entries given by $\left.G_{k l}=\delta_{k l}\left(\sum_{m=1}^{N} 1 / Z_{k m}\right)+\left(\delta_{k l}-1\right) / Z_{k l}\right], \mathbf{F}^{(s, t)}$ is the inflow/outflow vector (with non-zero entries only at node $s, F$, and at node $t,-F$ ), and $\mathbf{V}^{(s, t)}$ is the voltage potential at each node of the network. Properties of the weighted Laplacian matrix $\mathbf{G}$ are dealt in Appendix A.

\subsection{The analytical solutions}

We are deriving two main analytical results in this work. An expression for the DC/AC currents flowing through each edge of the network (as function of the location of the source and sink nodes and the net inflow magnitude) and the equivalent impedance between any two nodes of the network. Both results are expressed in terms of the eigenvalues and eigenvectors of the Laplacian matrix $\mathbf{G}$ of equation (5), and are found from the inversion of $\mathbf{G}$ (see Appendix B for details on the inversion of $\mathbf{G}$ ).

We find that after the inversion of $\mathbf{G}$ the voltage difference between any two nodes of the circuit is given by

$$
\Delta V_{k l}^{(s, t)}=F \sum_{n=1}^{N-1} \frac{\left(\left[\mathbf{v}_{n}\right]_{k}-\left[\mathbf{v}_{n}\right]_{l}\right)}{\lambda_{n}(\mathbf{G})}\left(\left[\mathbf{v}_{n}\right]_{s}^{\star}-\left[\mathbf{v}_{n}\right]_{t}^{\star}\right),
$$

where $\lambda_{n}(\mathbf{G})$ is the complex $n$-th eigenvalue of Laplacian $\mathbf{G}$ (with $n=0,1, \ldots, N-1$ and $\lambda_{0}(\mathbf{G})=0[2]$ ) and $\left[\mathbf{v}_{n}\right]_{k}$ is the corresponding $n$-th eigenvector $k$ coordinate (with $k=1, \ldots, N)$. With the exception of $F$ (assuming the phase difference between the net input and output flows is null, which guarantees global charge conservation), the remaining quantities are complex numbers, hence, they have an amplitude and a phase, and the $\star$ indicates complex conjugation. Thus,

$$
\Delta V_{k l}^{(s, t)}=F \sum_{n=1}^{N-1}\left[\alpha_{k l}^{(s t)}(n)+j \beta_{k l}^{(s t)}(n)\right] \frac{e^{j \phi_{k l}^{(s t)}}}{\lambda_{n}(\mathbf{G})}
$$

where $\alpha_{k l}^{(s t)}(n)\left[\beta_{k l}^{(s t)}(n)\right]$ is the real [imaginary] part of the product $\left(\left[\mathbf{v}_{n}\right]_{k}-\right.$ $\left.\left[\mathbf{v}_{n}\right]_{l}\right)\left(\left[\mathbf{v}_{n}\right]_{s}^{\star}-\left[\mathbf{v}_{n}\right]_{t}^{\star}\right)$ and the phases $\phi_{k l}^{(s t)}$ are

$$
\phi_{k l}^{(s t)}=\frac{-\alpha_{k l}^{(s t)}(n) \lambda_{n}\left(\mathbf{G}_{I}\right)+\beta_{k l}^{(s t)}(n) \lambda_{n}\left(\mathbf{G}_{R}\right)}{\alpha_{k l}^{(s t)}(n) \lambda_{n}\left(\mathbf{G}_{R}\right)+\beta_{k l}^{(s t)}(n) \lambda_{n}\left(\mathbf{G}_{I}\right)},
$$

with $\lambda_{n}\left(\mathbf{G}_{R}\right)\left[\lambda_{n}\left(\mathbf{G}_{I}\right)\right]$ being the $n$-th eigenvalue of the real [imaginary] part of the Laplacian matrix $\mathbf{G}$ (details on the properties of these eigenvalues are provided in Appendix A). 
Our first main analytical result is the current passing through the $k l$-edge, namely,

$$
I_{k l}^{(s, t)}=\frac{F}{Z_{k l}} \sum_{n=1}^{N-1}\left[\alpha_{k l}^{(s t)}(n)+j \beta_{k l}^{(s t)}(n)\right] \frac{e^{j \phi_{k l}^{(s t)}}}{\lambda_{n}(\mathbf{G})},
$$

where $Z_{k l}=\left|Z_{k l}\right| e^{j \varphi_{k l}}, \varphi_{k l}$ being the impedance phase value. This phase also corresponds to the phase difference between the voltage drop $\Delta V_{k l}^{(s, t)}$ between nodes $k$ and $l$ and the current $I_{k l}^{(s, t)}$ at the $k l$-edge.

Setting the source at node $k$ and the sink at node $l$, equation (6) results in

$$
\Delta V_{k l}^{(k, l)}=F \sum_{n=1}^{N-1} \frac{\left|\left[\mathbf{v}_{n}\right]_{k}-\left[\mathbf{v}_{n}\right]_{l}\right|^{2}}{\lambda_{n}(\mathbf{G})}
$$

and the second main analytical result is derived, i.e.,

$$
Z_{k l}^{(e q)}=\sum_{n=1}^{N-1} \frac{\left|\left[\mathbf{v}_{n}\right]_{k}-\left[\mathbf{v}_{n}\right]_{l}\right|^{2}}{\lambda_{n}(\mathbf{G})}
$$

$Z_{k l}^{(e q)}$ is the effective weight that all edges linking node $k$ with $l$ weigh $[5,6,15]$, namely, the equivalent impedance. Its value is identical to the ones obtained by using Green functions [14] or Circuit Theory analysis [1,17]. For example, if all series and parallel impedances between nodes $k$ and $l$ are added, then the final value is equal to the one that is found from equation (11).

\subsection{Practical examples}

Here we show how to relate the solution in equation (9) to the currents in the edges of a network-like circuit for given input/output nodes with the currents in the edges of a closed circuit with a single voltage generator. As it is known, solutions for closed linear circuits with given boundary voltage values correspond to finding solutions of the Laplace equation without sources, which are widely known. Hence, finding a relationship between equation (9) with the closed circuit currents, in our case, is equivalent to finding a relationship between topological invariant properties of the network structure (impedances) with Laplace boundary problem solutions.

For a single source-sink problem (like the one depicted in Fig. 2a) to be transformed to a Laplace problem (Fig. 2b), the source and sink nodes $(S$ and $T$ ) are plugged into a voltage generator. The known data from the source-sink problem is the fixed input net current $F$, which the generator will need to provide to the network circuit between nodes $S$ and $T$. Hence, the voltage requirement for the generator to supply is: $\epsilon_{s t}=V_{s}-V_{t}=\rho_{s t} F$, where $\rho_{s t}$ is the equivalent resistance between the nodes $S$ and $T$ of the known network circuit structure (dashed square in Fig. 2b). The equivalent resistance is found from $[3,5,6]$

$$
\rho_{s t}=\sum_{n=1}^{N-1} \frac{1}{\lambda_{n}}\left(\left[\mathbf{v}_{n}\right]_{s}-\left[\mathbf{v}_{n}\right]_{t}\right)^{2},
$$

where $\lambda_{n}\left(\mathbf{v}_{n}\right)$ is the real $n$-th eigenvalue (eigenvector) of the resistor network Laplacian matrix (which is also positively defined because its entries are all real valued). Equation (12) is the resistive version of equation (11). Then, the corresponding identical Laplace problem to the single source-sink pair of nodes is solved for the border 
(a)

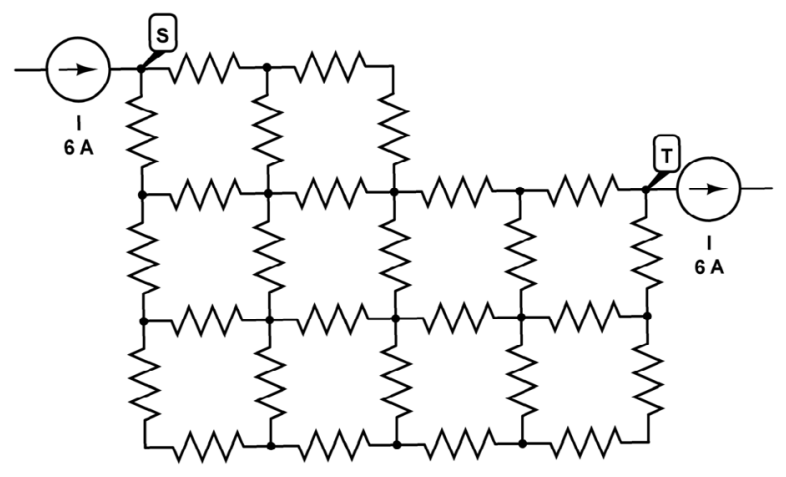

(b)

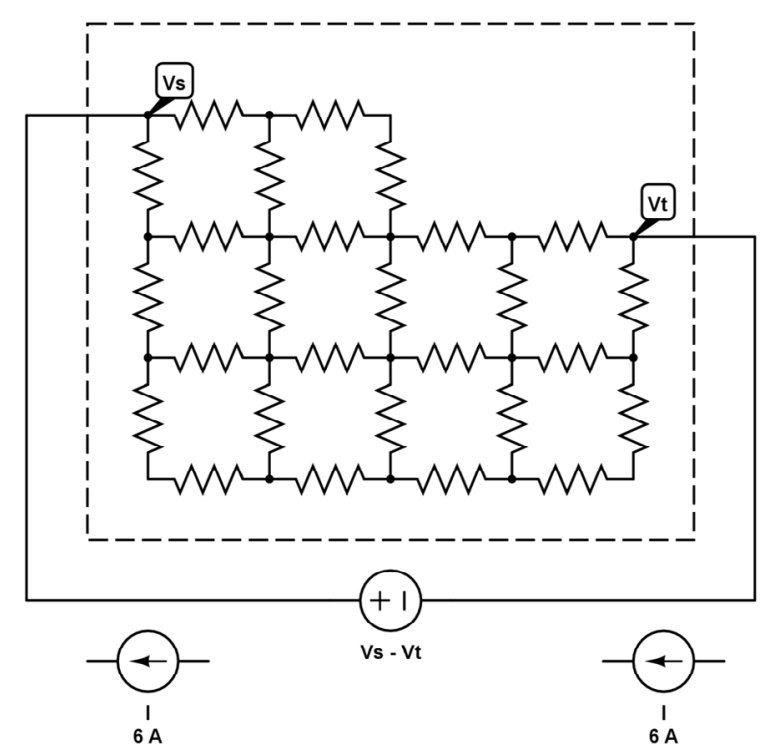

Fig. 2. Panel (a) shows a schematic representation of a resistor network with a single input current of $I=6 A$ at a source node $S$ and a single output current of $I=6 A$ at a sink node $T$. Panel (b) shows the same resistor network but with a voltage generator and a resistive line connecting nodes $S$ and $T$ such that the inflow (outflow) at node $S(T)$ equals $I$.

conditions corresponding to a voltage generator supplying a constant voltage difference $\epsilon_{s t}$ between nodes $S$ and $T$ of magnitude

$$
\epsilon_{s t}=\rho_{s t}(\mathcal{G}) F,
$$

where $\mathcal{G}=\{\mathcal{V}, \mathcal{E}\}$ is the node and edge set which define the circuit network. Equation (13) establishes a direct relationship between solving Laplace problems in circuits with transportation problems. Consequently, this relationship increases the importance of our voltage solution in terms of the Laplacian matrix spectral properties.

As another practical example, we compare our solutions for a square-like resistor network with equal edges with the solutions found from linear Circuit Theory analysis. 
We index the nodes in the square as $s, a, t, b$ in clockwise direction and the edge's resistances as $R_{s a}=R_{a t}=R_{s b}=R_{b t}=R$. Setting an input (output) source (sink) at node $s(t)$ with magnitude $F_{s}^{(s t)}=I\left(F_{t}^{(s t)}=-I\right)$, nodal analysis gives the following results for the edge currents that this circuit system has: $I_{s a}=I_{s b}=I / 2$ and $I_{t a}=$ $I_{t b}=-I / 2$. The resultant equivalent resistance between nodes $s$ and $t$ is

$$
\rho_{s t}=\left(\frac{1}{R_{s a}+R_{a t}}+\frac{1}{R_{s b}+R_{b t}}\right)^{-1}=R .
$$

In our framework, we transform the resistor network into a topological problem, i.e., we analyse the Laplacian matrix G of the network. Applying Ohm's and Kirchhoff's laws, a square-like resistor network has the following conductance matrix $\mathbf{G}$ [see Eq. (5)]

$$
\mathbf{G}=\frac{1}{R}\left(\begin{array}{cccc}
2 & -1 & -1 & 0 \\
-1 & 2 & 0 & -1 \\
-1 & 0 & 2 & -1 \\
0 & -1 & -1 & 2
\end{array}\right)=\frac{1}{R} \mathbf{L}
$$

where the first column/row corresponds to node $s$, then node $a$, node $b$, and finally node $t$. The eigenvalues of $\mathbf{G}$ are $\lambda_{0}=0, \lambda_{1}=\lambda_{2}=2 / R$, and $\lambda_{3}=4 / R$, and the eigenvectors are $\mathbf{v}_{0}=\frac{1}{2}(1,1,1,1)^{T}, \mathbf{v}_{1}=\frac{1}{\sqrt{2}}(0,-1,1,0)^{T}, \mathbf{v}_{2}=\frac{1}{\sqrt{2}}(1,0,0,-1)^{T}$, and $\mathbf{v}_{3}=\frac{1}{2}(-1,1,1,-1)^{T}$. Thus, using equation (6) for the edge current between nodes $s$ and $a$, we have

$$
I_{s a}^{(s t)}=\frac{\Delta V_{s a}^{(s t)}}{R}=\sum_{n=1}^{3}\left(\left[\mathbf{v}_{n}\right]_{s}-\left[\mathbf{v}_{n}\right]_{a}\right) \frac{I}{R \lambda_{n}}\left(\left[\mathbf{v}_{n}\right]_{s}-\left[\mathbf{v}_{n}\right]_{t}\right),
$$

that results in

$$
I_{s a}^{(s t)}=I\left(\left[\mathbf{v}_{2}\right]_{s}-\left[\mathbf{v}_{2}\right]_{a}\right) \frac{1}{2}\left(\left[\mathbf{v}_{2}\right]_{s}-\left[\mathbf{v}_{2}\right]_{t}\right)=\frac{I}{2},
$$

where the other eigenvector modes in the sum are cancelled or have null coordinates. Similarly, the remaining edge currents are calculated and found identical to the ones from nodal analysis. Moreover, the equivalent resistance for nodes $s$ and $t$ using equation (12) is

$$
\rho_{s t}=\frac{\left(\left[\mathbf{v}_{2}\right]_{s}-\left[\mathbf{v}_{2}\right]_{t}\right)^{2}}{2 / R}=R,
$$

which again, is identical to the one in equation (14).

We can extend this problem easily for the case where the square circuit has equal impedances $Z=R+j \omega L\left[1-\left(\omega_{0}^{2} / \omega\right)^{2}\right]$ in every edge, where $\omega_{0}^{2}=1 / L C$ is the characteristic frequency of each edge and $\omega$ is the input frequency $\left(F_{s}^{(s t)}(T)=I e^{j \omega T}=\right.$ $F_{t}^{(s t)}(T)$, for every time $\left.T\right)$. Then, the admittance Laplacian matrix entries from equation (5) are given by

$$
G_{k l}=\delta_{k l}\left(\sum_{m=1}^{4} \frac{1}{Z_{k m}}\right)-\frac{\left(\delta_{k l}-1\right)}{Z_{k l}} .
$$

The inverse of the impedance (admittance) is given by

$$
\frac{1}{Z_{k l}}=A_{k l} \frac{e^{-j \varphi}}{|Z|}
$$


where $A_{k l}=1$ if node $k$ is connected to node $l, A_{k l}=0$ otherwise, and $\tan (\varphi)=$ $L\left(\omega^{2}-\omega_{0}^{2}\right) / R \omega$. The resultant admittance Laplacian matrix in this case is

$$
\mathbf{G}=\mathbf{G}_{R}+j \mathbf{G}_{I}=\frac{e^{-j \varphi}}{|Z|} \mathbf{L}=\frac{\mathbf{L}}{Z},
$$

with $\mathbf{G}_{R}\left(\mathbf{G}_{I}\right)$ being the real (imaginary) part of the entries in equation (18) and $\mathbf{L}$ the Laplacian matrix from equation (15). Consequently, the eigenvalues of $\mathbf{G}$ are simply the eigenvalues of $\mathbf{L}$ divided by the impedance $Z$ and these matrices share the same eigenvectors.

In this case (the square circuit with identical impedances for its edges), the AC current flowing between nodes $s$ and $a$ is

$$
\begin{gathered}
\frac{\Delta V_{s a}^{(s t)}}{Z_{s a}}=\frac{I e^{j \omega T}}{Z_{s a}}\left(\left[\mathbf{v}_{2}\right]_{s}-\left[\mathbf{v}_{2}\right]_{a}\right) \frac{|Z|}{2 e^{-j \varphi}}\left(\left[\mathbf{v}_{2}\right]_{s}-\left[\mathbf{v}_{2}\right]_{t}\right), \\
I_{s a}^{(s t)}(T)=\frac{I}{2} e^{j \omega T},
\end{gathered}
$$

which is the same result as in the equal resistances DC case [Eq. (16)] for the modulus because $Z_{s a}=Z$. Furthermore, the analogy is further seen when calculating the equivalent impedance between the source $(s)$ and sink $(t)$ nodes using equation (11). This results in

$$
Z_{s t}^{(e q)}=\frac{\left|\left[\mathbf{v}_{2}\right]_{s}-\left[\mathbf{v}_{2}\right]_{t}\right|^{2}}{2 / Z}=Z
$$

The solution is identical to the one that Circuit Theory derives and is in direct correlation with the DC problem as expected.

In more general scenarios, the relationship between the $\mathrm{DC}$ and $\mathrm{AC}$ circuit is not direct. In such situations, the complex entries of the Laplacian matrix for the AC case are not related to the DC Laplacian matrix. Hence, further assumptions need to be done to find analytical solutions. For instance, one could have to impose that the input frequency to be larger than the natural frequencies of the lines $\left(\omega>\omega_{k l}\right.$ for every edge), such that the imaginary part of the Laplacian be positive semi-defined (see Appendix A).

\section{Conclusions}

The approach we develop provides new analytical insights into the transmission flow problem and exhibits different features than other available solutions. Moreover, it provides a tool to achieve the voltage/current solutions and to analyse resonant behaviour in linear circuits. As a practical application, we relate these solutions to closed circuits where a voltage generator is present (instead of having open sources/sinks that feed current to the network) and solve a simple network where we can compare our solutions to the ones provided by solving directly Kirchhoff's equations. Our findings help to solve problems, where the input and output nodes change in time within the network, more effectively than classical Circuit Theory techniques.

All authors thank the Scottish University Physics Alliance (SUPA) support. NR also acknowledges de support of PEDECIBA, Uruguay. MSB acknowledges the support of EPSRC grant Reference EP/I032606/1. 


\section{Appendix A: Complex weighted Laplacian matrix characteristics}

The weighted Laplacian matrix of the network $\mathbf{G}$ with edge properties given by the symmetric line impedances $Z_{k l}=Z_{l k}$ has the following complex-valued entries

$$
G_{k l}=\delta_{k l}\left(\sum_{m=1}^{M} \frac{1}{Z_{l m}}\right)+\frac{\left(\delta_{k l}-1\right)}{Z_{k l}},
$$

hence,

$$
\sum_{l=1}^{N} G_{k l}=0, \forall k
$$

which is the first requirement for a Laplacian matrix: the zero-row-sum property.

The eigenspace of $\mathbf{G}$ is composed of a set of $N$ complex eigenvalues $\lambda_{n}$ and eigenvectors $\mathbf{v}_{n}$ with $n=0,1, \ldots, N-1$, such that

$$
\mathbf{G} \mathbf{v}_{n}=\lambda_{n} \mathbf{v}_{n}, \forall n
$$

thus,

$$
\mathbf{G} \mathbf{P}=\mathbf{P} \Lambda,
$$

where $\mathbf{P}=\left\{\mathbf{v}_{0}, \mathbf{v}_{1}, \ldots, \mathbf{v}_{N-1}\right\}$ is a unitary matrix $\left(\mathbf{I}=\mathbf{P}^{-1} \mathbf{P}=\mathbf{P}^{\star T} \mathbf{P}, \mathbf{I}\right.$ being the identity matrix) of eigenvectors and $\Lambda$ is a diagonal matrix of eigenvalues $\left(\Lambda_{k l}=\right.$ $\left.\delta_{k l} \lambda_{k-1}\right)$.

Due to equation (A.2), G has a null eigenvalue (referred to as $\lambda_{0}$ in the following) associated to the kernel vector $\mathbf{v}_{0}=\mathbf{1} / \sqrt{N}$, where $\mathbf{1}=(1, \ldots, 1)$. Using equation (A.2), $\mathbf{G} \mathbf{1}=\mathbf{0}=\lambda_{0} \mathbf{1}$. Hence, the kernel of the matrix (the space of eigenvectors associated to the null eigenvalues) is at least of dimension 1 and direct inversion of the matrix is impossible. This is the second property of a Laplacian matrix,

$$
\operatorname{det}(\mathbf{G})=\operatorname{det}\left(\mathbf{P} \Lambda \mathbf{P}^{-1}\right)=\operatorname{det}(\Lambda)=\prod_{n=0}^{N-1} \lambda_{n}=0,
$$

which implies that the rank of the matrix is strictly less than $N$.

The third property is that Laplacian matrices are positive semi-defined. In particular, for any column vector $\mathbf{x}$, the Dirichlet sum is such that

$$
\mathbf{x} \cdot \mathbf{G} \mathbf{x}=\frac{1}{2} \sum_{k=1}^{M} \sum_{l=1}^{N} W_{k l}\left(x_{k}-x_{l}\right)^{2} \geq 0,
$$

where "." is the inner product operation and $W_{k l}=1 / Z_{k l}$ is the weighted adjacency matrix of the circuit network. This inequality holds only if $W_{k l} \geq 0$ for all $k$ and $l$. As a consequence, it implies that all eigenvalues are non-negative, because $\mathbf{x}$ can be any of the $\mathbf{v}_{n}$ eigenvectors. In that case,

$$
0 \leq \mathbf{v}_{n} \cdot \mathbf{G} \mathbf{v}_{n}=\lambda_{n} \mathbf{v}_{n} \cdot \mathbf{v}_{n}=\lambda_{n}
$$

where the last equality is possible because of the unitary property of the eigenvectors $\left(\mathbf{v}_{n} \cdot \mathbf{v}_{m}=\sum_{k=1}^{N}\left[\mathbf{v}_{n}\right]_{k}\left[\mathbf{v}_{m}\right]_{k}^{\star}=\delta_{n m}\right)$. However, equation (A.5) is always valid only for Laplacian matrices with non-negative real entries. 
For complex entries, such as in $\mathbf{G}$, the inequality in equation (A.5) can be analysed by splitting the matrix $\mathbf{G}$ into a real $\left(\mathbf{G}_{R}\right)$ and an imaginary $\left(\mathbf{G}_{I}\right)$ part, i.e., $\mathbf{G}=$ $\mathbf{G}_{R}+j \mathbf{G}_{I}$, where

$$
\begin{aligned}
& \mathbf{G}_{R}=\delta_{k l}\left(\sum_{m=1}^{M} \frac{\cos \left(\varphi_{l m}\right)}{\left|Z_{l m}\right|}\right)+\frac{\left(\delta_{k l}-1\right) \cos \left(\varphi_{k l}\right)}{\left|Z_{k l}\right|}, \\
& \mathbf{G}_{I}=-\delta_{k l}\left(\sum_{m=1}^{M} \frac{\sin \left(\varphi_{l m}\right)}{\left|Z_{l m}\right|}\right)-\frac{\left(\delta_{k l}-1\right) \sin \left(\varphi_{k l}\right)}{\left|Z_{k l}\right|},
\end{aligned}
$$

and $Z_{k l}=\left|Z_{k l}\right| e^{j \varphi_{k l}}$. The Laplacian matrix $\mathbf{G}_{R}$ contains the information of the network resistive part and the Laplacian matrix $\mathbf{G}_{I}$ contains the information of the network reactive part. In other words, the dissipative and the resonant structure of the circuit, respectively.

In order to have a positive (or negative) semi-defined Laplacian matrix, the weighted adjacency matrix elements need to be positive (or negative) for all pairs of nodes. For example, for the real part, if $W_{k l}^{(R)}=\cos \left(\varphi_{k l}\right) /\left|Z_{k l}\right| \geq 0$, then $\mathbf{G}_{R}$ is positive semi-defined. Consequently, the validity of this property depends on the magnitude of the phases that the line impedances introduce. In particular, for lines that can be modelled by series $R L C$, the $W_{k l}^{(R)} \geq 0$ for every $k l$-edge, hence, $\mathbf{G}_{R}$ has a non-negative spectra of eigenvalues. However, in such a case, the sign of $W_{k l}^{(I)}=\sin \left(\varphi_{k l}\right) /\left|Z_{k l}\right|$ depends on the input/output frequency [see Eq. (2)]. For $\omega<\omega_{k l}^{(L C)}$ for all $k l$-edges, $W_{k l}^{(I)} \geq 0$, thus $\mathbf{G}_{I}$ has a non-negative spectra of eigenvalues as $\mathbf{G}_{R}$. For $\omega>\omega_{k l}^{(L C)}, W_{k l}^{(I)} \leq 0$, thus, the opposite happens.

In general, a rule of thumb for knowing the character of the spectra of the matrix $\mathbf{G}$ is missing (the elements are complex and are unbounded, namely, the elements can be outside the complex-plane positive-quadrant). However, the unitary property of the associated eigenvectors set, $\left\{\mathbf{v}_{0}, \mathbf{v}_{1}, \ldots, \mathbf{v}_{N-1}\right\}$, is always valid, meaning that

$$
\mathbf{v}_{n} \cdot \mathbf{v}_{m}=\sum_{k=1}^{N}\left[\mathbf{v}_{n}\right]_{k}\left[\mathbf{v}_{m}\right]_{k}^{\star}=\delta_{n m},
$$

and

$$
\left[\mathbf{P}^{-1} \mathbf{P}\right]_{k l}=\sum_{n=0}^{N-1}\left[\mathbf{v}_{n}\right]_{k}\left[\mathbf{v}_{n}\right]_{l}^{\star}=\delta_{k l} .
$$

Equation (A.8) is the verification that the eigenvector set is composed solely by linearly independent vectors. Equation (A.9) is the completeness property, and it is the verification that the set is also a generating set. Hence, it conforms a basis of the linear functions that operate over the set of nodes.

\section{Appendix B: Inversion of the Laplacian matrix and the node voltage potential solutions}

Due to the existence of the null eigenvalue in any Laplacian matrix, the inverse is ill defined. We overcome this problem by means of a translation in equation (5) and the removal of the kernel from the eigenvector base. The process results in what is known as the Moore-Penrose inverse Laplacian matrix [23]. Namely,

$$
\mathbf{G} \mathbf{V}^{(s t)}+\frac{\mathbf{J}}{N} \mathbf{V}^{(s t)}=\mathbf{F}^{(s t)}+\boldsymbol{e}^{(s t)}
$$


where all entries of matrix $\mathbf{J}$ are equal to unity $\left(J_{k l}=1\right)$ and $e_{i}^{(s t)}=\frac{1}{N} \sum_{k} V_{k}^{(s t)}$, with $i=1, \ldots, N$, is the resultant vector of the product between $\mathbf{J} / N$ and $\mathbf{V}^{(s t)}$.

From equation (A.3) we can write the elements of $\mathbf{G}$ in terms of its complex eigenvalues and eigenvectors as

$$
G_{k l}=\sum_{n=1}^{N-1}\left[\mathbf{v}_{n}\right]_{k} \lambda_{n}\left[\mathbf{v}_{n}\right]_{l}^{\star},
$$

where the term corresponding to $n=0$ has been removed because $\lambda_{0}=0$. Similarly, we define the following matrix $\mathbf{X}$ entries by

$$
X_{k l}=\sum_{n=1}^{N-1}\left[\mathbf{v}_{n}\right]_{k} \frac{1}{\lambda_{n}}\left[\mathbf{v}_{n}\right]_{l}^{\star} .
$$

Here we show that $\mathbf{X}+\mathbf{J} / N$ is the inverse matrix of $\mathbf{G}+\mathbf{J} / N$. In general, $\mathbf{G}$ is a matrix that represents the edge admittances, whereas $\mathbf{X}$ represents the edge impedances. First, we note that $\mathbf{J}^{2}=N \mathbf{J}$, hence, $\mathbf{J}^{2} / N^{2}=\mathbf{J} / N$. Then, we observe that $\mathbf{G} \mathbf{J}=\mathbf{0}$ because of the zero-row-sum property. Similarly, $\mathbf{X} \mathbf{J}=\mathbf{0}$. This is seen from,

$$
\sum_{l=1}^{N} X_{k l} J_{l m}=\sum_{l=1}^{N} X_{k l}=\sum_{n=1}^{N-1}\left[\mathbf{v}_{n}\right]_{k} \frac{1}{\lambda_{n}} \sum_{l=1}^{N}\left[\mathbf{v}_{n}\right]_{l}^{\star} .
$$

However, as equation (A.8) holds for every eigenvector of $\mathbf{G}$, in particular, $\mathbf{v}_{0}$. $\mathbf{v}_{m}=\mathbf{1} \cdot \mathbf{v}_{m} / \sqrt{N}=\delta_{0 m}$, then $\sum_{l=1}^{N}\left[\mathbf{v}_{n}\right]_{l}^{\star}=0$ for every spanning eigenvector $(n \neq 0)$. Finally,

$$
\begin{aligned}
{[\mathbf{X ~ G}]_{k p} } & =\sum_{l=1}^{N}\left(\sum_{n=1}^{N-1}\left[\mathbf{v}_{n}\right]_{k} \frac{1}{\lambda_{n}}\left[\mathbf{v}_{n}\right]_{l}^{\star} \sum_{m=1}^{N-1}\left[\mathbf{v}_{m}\right]_{l} \lambda_{m}\left[\mathbf{v}_{m}\right]_{p}^{\star}\right) \\
& =\sum_{n=1}^{N-1} \sum_{m=1}^{N-1}\left[\mathbf{v}_{n}\right]_{k} \frac{1}{\lambda_{n}}\left(\sum_{l=1}^{N}\left[\mathbf{v}_{n}\right]_{l}^{\star}\left[\mathbf{v}_{m}\right]_{l}\right) \lambda_{m}\left[\mathbf{v}_{m}\right]_{p}^{\star},
\end{aligned}
$$

where, using equation (A.8), results in

$$
[\mathbf{X ~ G}]_{k p}=\sum_{n=1}^{N-1}\left[\mathbf{v}_{n}\right]_{k}\left[\mathbf{v}_{n}\right]_{p}^{\star} .
$$

Now, observing that equation (A.9) can be written as

$$
\frac{1}{N}+\sum_{n=1}^{N-1}\left[\mathbf{v}_{n}\right]_{k}\left[\mathbf{v}_{n}\right]_{p}^{\star}=\delta_{k p}
$$

Equation (B.4) is further simplified to

$$
[\mathbf{X ~ G}]_{k p}=\delta_{k p}-\frac{1}{N}=[\mathbf{I}]_{k p}-\frac{[\mathbf{J}]_{k p}}{N} .
$$

Consequently, we have shown that

$$
\left(\mathbf{X}+\frac{\mathbf{J}}{N}\right)\left(\mathbf{G}+\frac{\mathbf{J}}{N}\right)=\mathbf{I} .
$$


Returning to equation (B.1), and using equation (B.6), we obtain the voltage potentials at each node

$$
\mathbf{V}^{(s t)}=\left(\mathbf{X}+\frac{\mathbf{J}}{N}\right) \mathbf{F}^{(s t)}+\boldsymbol{e}^{(s t)}, 0
$$

where we use that $\mathbf{X} \boldsymbol{e}^{(s t)}=\mathbf{0}$ and $\mathbf{J} \boldsymbol{e}^{(s t)}=N \boldsymbol{e}^{(s t)}$. If global conservation of charge is granted, namely, if the input current equals the output current in phase and magnitude, then, $\mathbf{J} \mathbf{F}^{(s t)}=\mathbf{0}$. Otherwise, $\mathbf{J} \mathbf{F}^{(s t)}$ is a vector with all the elements equal to the magnitude and/or phase difference between the input and output net currents [see Eq. (3)]. We note that the role of $\boldsymbol{e}^{(s t)}$ in equation (B.7) is to add an arbitrary constant to the node voltage potential. This is easily interpreted as the arbitrary energy reference point. Such arbitrary value is eliminated once voltage differences are calculated. Moreover, voltage differences eliminate also the possible constant value given by $\mathbf{J} \mathbf{F}^{(s t)}$. Consequently, the voltage difference between two arbitrary nodes $k$ and $l$ in the network is given by

$$
\Delta V_{k l}^{(s t)}=V_{k}^{(s t)}-V_{l}^{(s t)}=\left[\mathbf{X} \mathbf{F}^{(s t)}\right]_{k}-\left[\mathbf{X} \mathbf{F}^{(s t)}\right]_{l}
$$

Thus,

$$
\begin{aligned}
\Delta V_{k l}^{(s t)} & =F^{i n}\left([\mathbf{X}]_{k s}-[\mathbf{X}]_{l s}\right)-F^{o u t}\left([\mathbf{X}]_{k t}-[\mathbf{X}]_{l t}\right) \\
& =F\left[\left([\mathbf{X}]_{k s}-[\mathbf{X}]_{l s}\right)-\left([\mathbf{X}]_{k t}-[\mathbf{X}]_{l t}\right)\right] \\
& =F\left[\sum_{n=1}^{N-1}\left(\left[\mathbf{v}_{n}\right]_{k}-\left[\mathbf{v}_{n}\right]_{l}\right) \frac{1}{\lambda_{n}}\left(\left[\mathbf{v}_{n}\right]_{s}^{\star}-\left[\mathbf{v}_{n}\right]_{t}^{\star}\right)\right] .
\end{aligned}
$$

\section{Appendix C: Many input/output flows and the relationship to voltage generators}

In order to analyse how equation (B.8) changes when many sources and sinks are present, we need to rewrite equation (3) to include the new sources of inflow and sinks of outflow. Thus, in general, the net current at a node is

$$
\sum_{l=1}^{N} I_{k l}^{\left(\mathcal{V}_{s}, \mathcal{V}_{t}\right)}=F\left(\sum_{s \in \mathcal{V}_{s}} a_{s} \delta_{k s}-\sum_{t \in \mathcal{V}_{t}} b_{t} \delta_{k t}\right)
$$

where $\mathcal{V}_{s}\left(\mathcal{V}_{t}\right)$ is the set of nodes that act as sources (sinks) and $a_{s}\left(b_{s}\right)$ is the fraction of the total inflow (outflow) $F$ that goes through node $s \in \mathcal{V}_{s}\left(t \in \mathcal{V}_{t}\right)$, namely, $\sum_{s \in \mathcal{V}_{s}} a_{s}=1\left(\sum_{t \in \mathcal{V}_{t}} b_{t}=1\right)$. Consequently, global conservation of charge is granted. Substituting equation (C.1) into equation (B.8), the voltage difference between nodes $k$ and $l$ in the circuit network with multiple sources and sinks is

$$
\Delta V_{k l}^{\left(\mathcal{V}_{s}, \mathcal{V}_{t}\right)}=F\left[\sum_{n=1}^{N-1}\left(\left[\mathbf{v}_{n}\right]_{k}-\left[\mathbf{v}_{n}\right]_{l}\right) \times \frac{1}{\lambda_{n}} \times\left(\sum_{s \in \mathcal{V}_{s}} a_{s}\left[\mathbf{v}_{n}\right]_{s}^{\star}-\sum_{t \in \mathcal{V}_{t}} b_{t}\left[\mathbf{v}_{n}\right]_{t}^{\star}\right)\right]
$$

When multiple sources and sinks exist (e.g., Fig. C.1a), then the transformation of the problem to a closed circuit problem requires the inclusion of a single super source $S$ node and super sink node $T$ need to be created (Fig. C.1b). All original source 
(a)

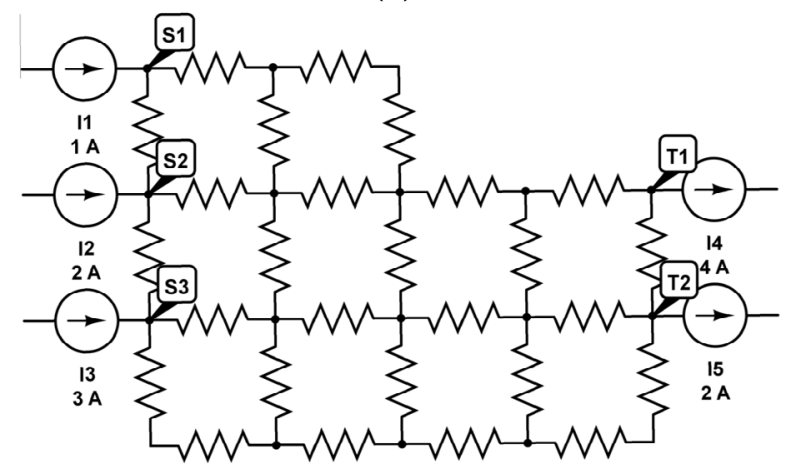

(b)

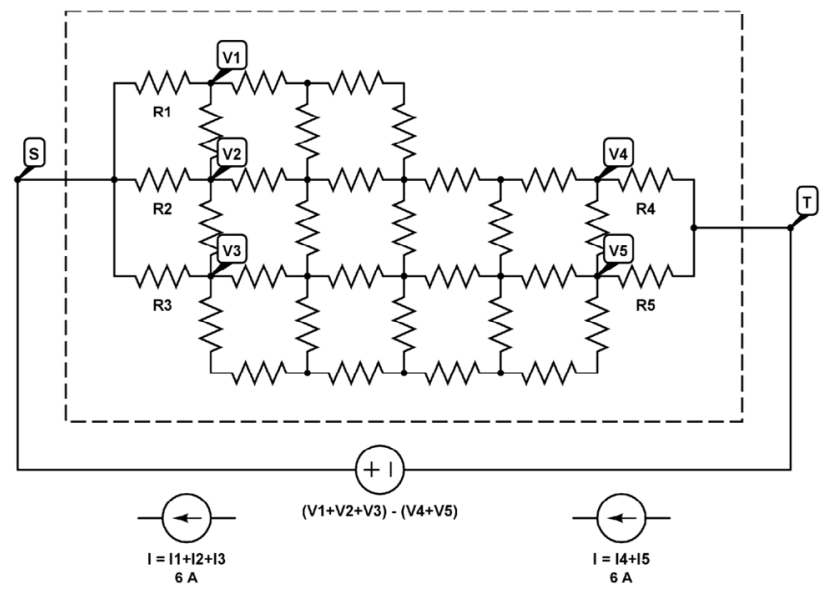

Fig. C.1. Panel (a) shows a schematic representation of a resistor network with many input $\left(I_{1}, I_{2}, I_{3}\right.$, nodes $\left.S_{1}, S_{2}, S_{3}\right)$ and output $\left(I_{4}, I_{5}\right.$, nodes $\left.T_{1}, T_{2}\right)$ currents. Panel (b) shows the same resistor network but as a closed system containing a voltage generator and a new resistor set. These supply the input (output) currents at nodes $S_{1}, S_{2}, S_{3}\left(T_{1}, T_{2}\right)$ via the new resistors $\left(R_{1}, R_{2}, \ldots\right)$ with an identical magnitude as in panel (a).

(sink) nodes are then connected to the new super source (sink) node that provides the total input (output) that the multiple sources (sinks) were feeding (consuming) in the original system $\mathcal{G}$, namely, $I(-I)$. Consequently, the multiple source-sink configuration in $\mathcal{G}$ is transformed into a single $S$ - $T$ pair configuration of a new network $\tilde{\mathcal{G}}$ that has 2 nodes more than the original network $\mathcal{G}$. In such conditions, the former process enables to analyse the new network setting by means of a single generator that connects these two new nodes. In other words, once a super source $S(\operatorname{sink} T)$ that connects to all the original sources $S_{i} \in \mathcal{V}_{s} \subset \mathcal{V}\left(\operatorname{sinks} T_{j} \in \mathcal{V}_{t} \subset \mathcal{V}\right)$ is defined, then a Laplace problem can be defined by setting a voltage generator providing

$$
\tilde{\epsilon}_{S T}=\rho_{S T}(\tilde{\mathcal{G}}) I
$$

where the equivalent resistance $\rho_{S T}(\tilde{\mathcal{G}})$ between the super source and super sink is unknown. This is because the impedance (resistance) values for the new edge connections between the multiple sources $S_{i}\left(\operatorname{sinks} T_{j}\right)$ to the super source are unknown. Moroever, they have to be set such that the current entering the network circuit 
through the old multiple sources (sinks) is identical to the one the particular source (sink) supplies (consumes), e.g., as in Figure C.1b.

In order to determine the impedances (resistances) of the edges connecting the super source (sink) to the multiple source (sink) nodes, we observe that:

$$
\left\{\begin{array}{l}
V_{S}-V_{i}^{\text {in }}=R_{i}^{\text {in }} a_{i} I, \quad \text { if } i \in\{\mathcal{S}\} \\
V_{j}^{\text {out }}-V_{T}=R_{j}^{\text {out }} b_{j} I, \text { if } j \in\{\mathcal{T}\}
\end{array},\right.
$$

where neither the voltages nor the resistances are known. Nevertheless, the voltages of the super nodes fulfil equation (C.2), thus, we can arbitrarily set the unknown resistances, $R_{i}^{i n}$ and $R_{j}^{\text {out }}$, for the new edges to unity. Consequently, $\rho_{S T}(\tilde{\mathcal{G}})$ can be derived and the node voltages for each of the multiple sources and sinks calculated, i.e.,

$$
\left\{\begin{array}{l}
V_{i}^{\text {in }}=V_{S}-1 a_{i} I=\left(\rho_{S T}-a_{i}\right) I+V_{T}, \\
V_{j}^{\text {out }}=V_{T}+1 b_{j} I=\left(-\rho_{S T}+b_{i}\right) I+V_{S},
\end{array}\right.
$$

where we use the fact that $\tilde{\epsilon}_{S T}=V_{S}-V_{T}$ [Eq. (C.2)] and note that both experssions necessarily have always an arbitrary constant $\left(V_{S}\right.$ and $\left.V_{T}\right)$.

\section{References}

1. P.R. Clayton, Fundamentals of electric circuit analysis (Wiley, 2001)

2. F.R.K. Chung, Spectral Graph Theory (American Mathematical Soc. and CBMS, 1997), Vol. 92

3. D. Randall, Rapidly mixing Markov chains with applications in computer science, physics, Comput. Sci. Eng. 8, 30 (2006)

4. M.E.J. Newman, M. Girvan, Finding, evaluating community structure in networks, Phys. Rev. E 69, 026113 (2004)

5. N. Rubido, C. Grebogi, M.S. Baptista, Structure, function in flow networks, Europhys. Lett. 101, 68001 (2013)

6. N. Rubido, C. Grebogi, M.S. Baptista, Resiliently evolving supply-demand networks, Phys. Rev. E 89, 012801 (2014)

7. G.G. Batrouni, A. Hansen, Fracture in Three-Dimensional Fuse Networks, Phys. Rev. Lett. 80, 325 (1998)

8. E. Katifori, G.J. Szollosi, M.O. Magnasco, Damage, Fluctuations Induce Loops in Optimal Transport Networks, Phys. Rev. Lett. 104, 048704 (2010)

9. A. Cardillo, M. Zanin, J. Gómez-Gardeñes, M. Romance, A.J. García del Amo, S. Boccaletti, Modeling the multi-layer nature of the European Air Transport Network: Resilience, passengers re-scheduling under random failures, Eur. Phys. J. Special Topics 215, 23 (2013)

10. Z. Liu, S. Pang, S. Gong, P. Yang, Robot Path Planning in Impedance Networks, in Proc. of 6th World Congr. on Intell. Control and Automation 2, 9109 (2006)

11. M. Haenggi, Analogy between data networks, electronic networks, Electron. Lett. 38, $553(2002)$

12. A. Hajimiri, Generalized Time-, Transfer-Constant Circuit Analysis, IEEE Trans. Circuits Syst. I: Regular Papers, 57, 1105 (2010)

13. R. Jakushokas, E.G. Friedman, Power Network Optimization Based on Link Breaking Methodology, IEEE Trans. on very large scale Int. (VLSI) Syst. 21, 983 (2013)

14. J. Cserti, Application of the lattice Green's function for calculating the resistance of infinite networks of resistors, Am. J. Phys. 68, 896 (2000)

15. F.Y. Wu, Theory of resistor networks: the two-point resistance, J. Phys. A: Math. Gen. 37, 6653 (2004)

16. J. Zheng Jiang, M.C. Smith, Series-Parallel Six-Element Synthesis of Biquadratic Impedances, IEEE Trans. Circuits Syst. I: Regular Papers, 59, 2543 (2012) 
17. J.E. Brittain, Thévenin's theorem, IEEE Spectrum, 27, 42 (2002)

18. R.K. Ahuja, T.L. Magnanti, J.B. Orlin, Network Flows: Theory, Algorithms, and Applications (Prentice-Hall, 1993)

19. F.G.S. Silva, R.N.de Lima, R.C.S. Freire, C. Plett, A Switchless Multiband Impedance Matching Technique Based on Multiresonant Circuits, IEEE Trans. Circuits Syst. II: Express Briefs 60, 417 (2013)

20. M.G. Rosenblum, A.S. Pikovsky, J. Kurths, Phase Synchronization of Chaotic Oscillators, Phys. Rev. Lett. 76, 1804 (1996)

21. L.M. Pecora, T.L. Carroll, Master Stability Functions for Synchronized Coupled Systems, Phys. Rev. Lett. 80, 2109 (1998)

22. L. Huang, Q. Chen, Y.-C. Lai, L.M. Pecora, Generic behavior of master-stability functions in coupled nonlinear dynamical systems, Phys. Rev. E 80, 036204 (2009)

23. S.L. Campbell, C.D. Meyer, Generalized inverses of linear transformations (Society for industrial and applied Mathematics, 2009), Chap. 1

Open Access This is an Open Access article distributed under the terms of the Creative Commons Attribution License (http://creativecommons.org/licenses/by/4.0), which permits unrestricted use, distribution, and reproduction in any medium, provided the original work is properly cited. 